\title{
Characteristics Analysis of a Novel Segmental Rotor Axial Field Switched Reluctance Motor with Single Teeth Winding
}

\author{
Bo Wang ${ }^{*}$, Dong-Hee Lee*, Chee-Woo Lee ${ }^{* *}$, and Jin-Woo Ahn ${ }^{\dagger}$ \\ ${ }^{*}$ Department of Mechatronics Engineering, Kyungsung University, Busan, Korea \\ ${ }^{* *}$ Department of Electrical Engineering, Pusan National University, Busan, Korea
}

\begin{abstract}
A novel 12/10 axial field switched reluctance motor (AF-SRM) is proposed for cooling fan applications in this paper. Unlike from conventional structures, the proposed motor uses the axial field instead of the radio field, the rotor is constructed from a series of discrete segments, and the stator poles are constructed from two types of stator poles: exciting and auxiliary poles. This concept improves the torque capability of a previous design by reducing the copper volume, which leads to a higher efficiency. To verify the proposed structure, the finite element method (FEM) and Matlab-Simulink are employed to get characteristics of the proposed SRM. Finally, a prototype of the proposed motor was tested for characteristic comparisons.
\end{abstract}

Key words: Axial field SRM, High torque, Low copper loss, Segmental rotor, Single teeth stator

\section{INTRODUCTION}

The magnetic geometry of conventional radial field switched reluctance machines (SRMs) has been effectively fixed for over 20 years. The basic structure consists of a series of stator teeth or poles, magnetically connected together by a core back, and a series of rotor teeth, with the magnetic circuit completed by a rotor core back. With secure operation and a wide speed region, the conventional SRMs can be widely used in industrial applications.

However, SRMs possess several disadvantages, especially for low speed high torque and short shaft applications, where the traditional radial field cannot provide a better performance [1]. Meanwhile, the recently proposed short flux path SRMs with an E-core and a C-core are possible low-cost solutions for variable-speed applications [2]-[4]. With their short flux path, the distance of each exaction path is reduced. Meanwhile, the stator has lower core losses due to the absence of flux reversals in the stator core and a phase flux path that only traverses two-thirds of the back iron, which lowers the magneto motive

Manuscript received Apr. 23, 2014; accepted Jul. 31, 2014

Recommended for publication by Associate Editor Tae-Won Chun.

${ }^{\dagger}$ Corresponding Author: jwahn@ks.ac.kr

Tel: +82-51-663-4773, Kyungsung University

*Dept. of Mechatronics Engineering, Kyungsung University, Korea

${ }^{* *}$ Dept. of Electrical Engineering, Pusan National University, Korea force (MMF) requirements. The goal of the segmental rotor type SRMs [4], is to reduce the iron loss. The rotor cores are cut into segmental parts and embedded into the rotor body. In this way, the weight of the rotor will be reduced and along with the rotational inertia. More importantly, with the segmental structure, the flux path is reduced by almost one third when compared with the conventional full path SRMs, and during variable-speed applications, the advantages of this low loss will be highlighted [5].

For this project, a novel axial field SRM is proposed to replace the conventional $12 / 8$ SRM for the cooling fan application. The proposed motor should be able to provide better output torque performance while reducing the copper loss, which is used to enhance the capacity of heat dissipation. With these requirements in mind, an axial magnetic field disk type induction motor (DSIMs) is proposed. Unlike traditional radial magnetic field rotating machines, the stator and rotor cores of the DSIMs are laminated into disk shape and the two iron cores take relative positions in space [6-7]. Because of this special structure, the DSIMs can accept a higher current density before oversaturation, which can be used to provide a higher output torque and overload capacity. Fig.1 shows the conventional structure of the stator and rotor of the SRMs and DSIMs.

By combining the advantages of the SRMs and DSIMs, a novel axial field SRM with single teeth winding and segmental 

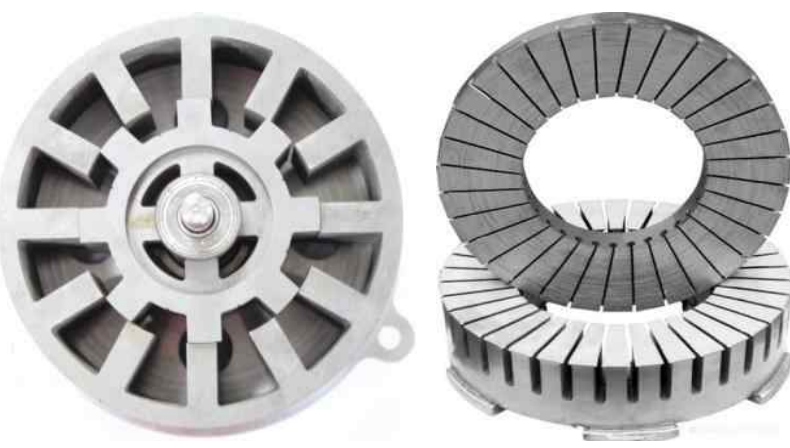

Fig. 1. Conventional structure of SRM and DSIM.

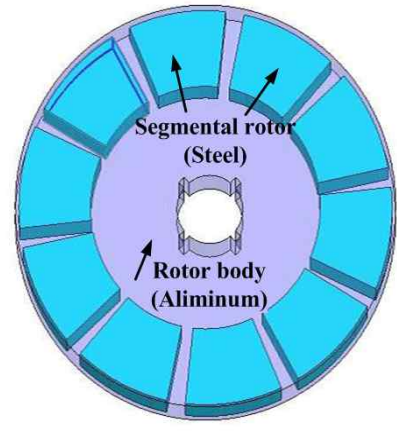

(a) Segmental rotor.

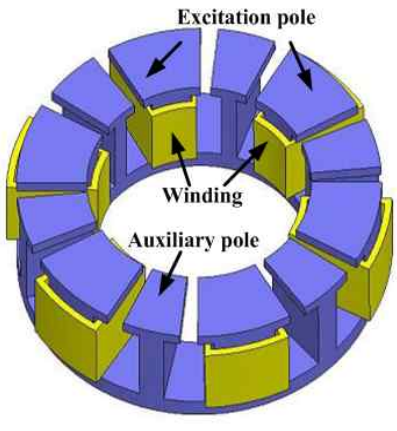

(b) Single teeth stator.
Fig. 2. 3D structure of the proposed design.

rotor is proposed. The stator and rotor cores are laminated into disk shape, and the stator teeth are formed into two groups, double width teeth-excitation, which are wound by the coils, standard width teeth-auxiliary, which are unwound but used to provide the circuit for the flux path. The rotor is made up of a series of segmental rotor blocks, which are embedded into the aluminum body. The characteristics of the proposed structure are analyzed, and the control scheme, simulation and experimental results are presented. When compared with previous studies, the proposed motor can provide a much better output performance with a low cost. Fig. 2 shows a 3D model of the proposed motor.

\section{Fundamental MAGNETIC DESIGN CONCEPTS}

This section presents the basic magnetic and design concepts of the axial field 12/10 SRM. Before progressing to a full rotating machine design, Fig.3 shows a rectilinear diagram of the multi-tooth structure SRM. The full pith windings are wound on the exaction pole at the aligned position. For this kind of conventional multi teeth type SRM, the machine had substantially longer end-windings, which reduced the electric loading and made it impractical for applications which combined a short lamination stack length with a large pole pitch.

Considering the cumbrous end-windings, in order to develop a three-phase design which is suitable for short shack SRMs, it is essential that the winding spans a single tooth to keep the

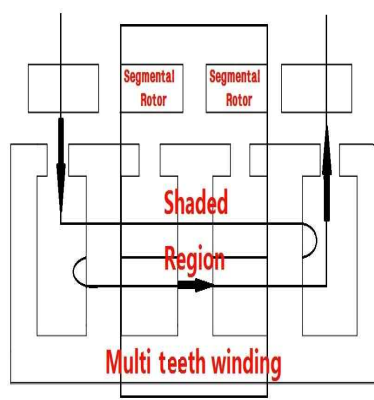

(a) Multi-teeth winding.

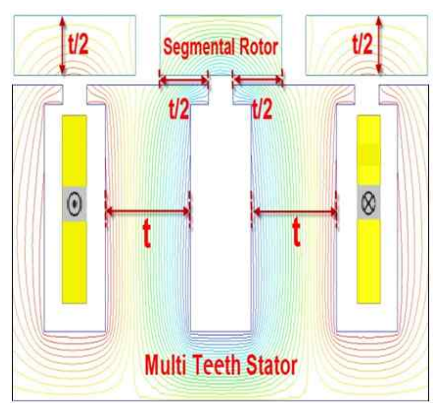

(b) Flux path at aligned position.
Fig. 3. Rectilinear diagram of multi tooth stator.

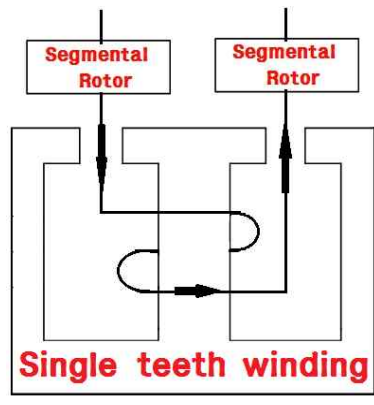

(a) Single-teeth winding.

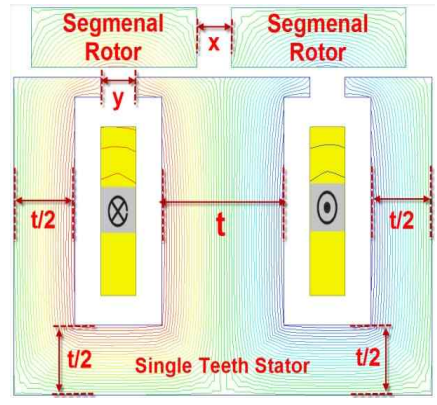

(b) Flux path at aligned position.
Fig. 4. Rectilinear diagram of single teeth stator.

end-winding shorter, whilst maintaining the condition where only the conductors of a single-phase occupy any one slot. As shown in Fig. 3(a) and 4(a), taking off the shaded region of the multi tooth and combining the rest of the part, will result in the "single teeth winding", which makes it so that the coils for one phase span just one stator. By this way, the rotor structure should only permit the adjoining teeth to be magnetically linked, and the magnetic flux can only enclose a single stator slot, in order to reduce the copper volume and to form a short flux path. The short flux can be used to reduce the iron loss of the rotor. The single tooth stator is twice the width of the previous pole. Meanwhile, one phase coil can span only one segmental rotor as shown in Fig. 4(b).

The single tooth topology is made up of two groups: double-width teeth, which are wound by the coils and standard-width teeth which are unwound. The unwound teeth are used to provide an overlap for the flux path which is produced by the wound teeth. The excitation of a single phase now excites the two adjacent slots and the phase permeance is the sum of the two slot permeances. The tooth pitch of the wound stator teeth must be equal to the rotor pole pitch, so that the permeance variation of these two slots with respect to the rotor position is in phase. For clarity a complete set of design rules for the single tooth structure design are given below:

(a) Only one phase winding is wound on one exaction pole, so that the adjacent stator can be wound on the same turnings to ensure the MMF of each phase, as shown in Fig. 4(b).

(b) The width of the gap between rotor segments isequal to 
TABLE I

Dimensions OF THE PROTOTYPE MOTOR

\begin{tabular}{cc}
\hline Parameters & $12 / 10$ AF-SRM \\
\hline Number of phases & 3 \\
Number of stator slots & 12 \\
Number of segmental rotors & 10 \\
Stator outside diameter(mm) & 104 \\
Rotor outside diameter(mm) & 106 \\
Stack axial length $(\mathrm{mm})$ & 36 \\
Air gap length $(\mathrm{mm})$ & 0.35 \\
Stator tooth width: & Double-width teeth: 30.25 \\
Tip of pole(degree) & Standard-width teeth: 18.5 \\
Stator tooth width: & Double-width teeth: 20 \\
Body of pole(degree) & Standard-width teeth: 8 \\
Segmental rotor width(degree) & 30.25 \\
Number of series turns/phase & 10 \\
\hline
\end{tabular}

that of the stator slot openings. This ensures that neither the rotor nor the stator contribute unnecessarily to the unaligned permeance, as shown in Fig. 4(b), $x=y$.

(c) The width of the unwounded pole $t / 2$ should be equal to the vertical height of the yoke of the stator $t / 2$, which is half the length of the wound pole $t$, to ensure that the flux density in the stator is uniform, as shown in Fig. 3(b).

(d) The radial depth of both the stator core back and the rotor segments is equal to the width of the unwound tooth since they only carry the flux of one unwound tooth, as shown in Fig. 3 (b).

The major dimensions of the prototype machine are shown in Table I.

\section{CHARACTERISTIC ANALYSIS OF THE AF-SRM}

The SRMs have very high nonlinear magnetization characteristics, so the 3D FEA is used to obtain the nonlinear magnetization data, to estimate the performance of the flux linkage, inductance, output torque, force and loss.

\section{A. Inductance Characteristics}

In most SRMs applications, saturation occurs, resulting in a nonlinear inductance and flux linkage. Since the proposed motor has 10 rotor poles, a rotation of $36^{\circ}$ from the aligned position to the un-aligned position is sufficient to obtain the inductance profile characteristic of one electrical cycle. The initial rotor position of the motor is at the un-aligned position and rotates in the counter clockwise direction. In this case, the inductance is at its highest and the magnetic reluctance of the flux is at its lowest. The inductance curves with different rotor positions are shown in Fig. 5 for various excitation currents. In addition, the flux linkage depends on the value of the inductance multiplied by the various corresponding currents.

\section{B. Output Torque Characteristics}

The SRMs are produced by the attraction of the rotor poles to the excited stator phase, depending on the reluctance

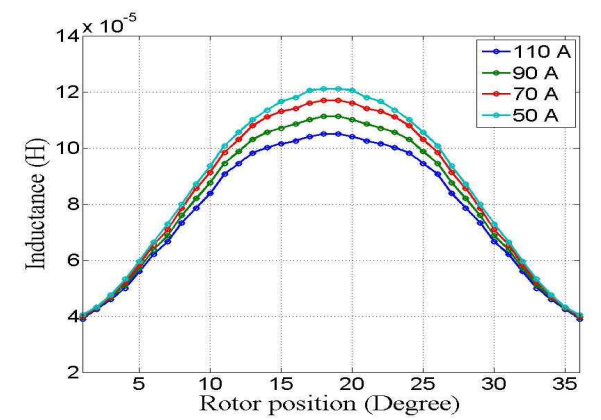

Fig. 5. Inductance profile of the proposed 12/10 SRM.

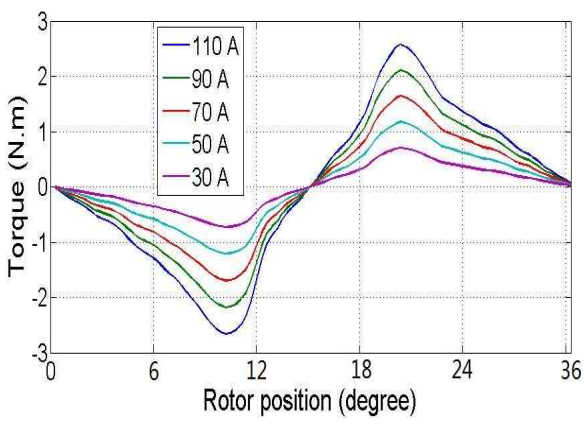

Fig. 6. Torque profile of the proposed 12/10 SRM.

principle. The phase torque depends on the square of the phase current and inductance. According to the above analysis, the change rate of the inductance changes rapidly from the aligned position to the unaligned position. Therefore, the output torque will get its peak value during the two regions. For the proposed design, each segment rotor is $30.25^{\circ}$. Hence, the torque curve is narrow when compared with the conventional type 12/8 SRM. However, the average torque of the proposed motor is much better than the conventional radio field 12/8 SRM. As can be seen in Table II, it is increased by $11.6 \%$. Fig. 6 shows the torque profile with various excited currents of the proposed AF-SRM. The shape of the torque curve for the proposed motor is similar to that of the conventional disk type induction motor. It is narrow and has a embossment at the aligned position.

\section{Axial Force with Uniform and Non-Uniform Air Gap}

There is an electromagnetic force in the axial direction between the stator and rotor caused by the axial field magnetic flux. Meanwhile, the axial force causes a vibration of the rotor during operating. For a uniform air gap which means that the air gaps by $\mathrm{D} 1=\mathrm{D} 2=\mathrm{D} 3=0.35 \mathrm{~mm}$, and for a non-uniform air gap, the air gap D1 is constant because of the mechanical strength of the shaft. However, for D2 and D3, one will be increased and the other one will be decreased. This is caused by the vibration. However, D2+D3 $=0.7 \mathrm{~mm}$ should be constant. As shown in Fig. 7, whether the air gap is uniform or not, the axial force will be reduced when the air gap is increased.

\section{Performance Comparison}



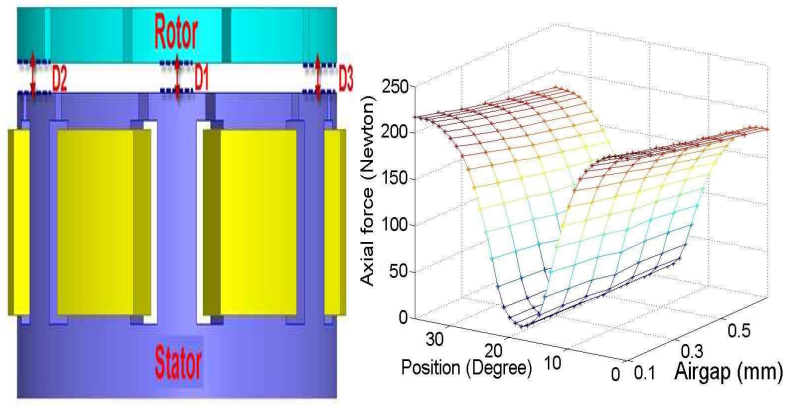

Fig. 7. Axial force with different air gaps.

TABLE II

COMPARISON WITH CONVENTIONAL 12/8 SRM

\begin{tabular}{ccc}
\hline Parameters & $12 / 8$ SRM & $12 / 10$ AF-SRM \\
\hline Number of phases & 3 & 3 \\
Number of stator poles & 12 & 12 \\
Number of rotor poles & 8 & 10 \\
Outside diameter (mm) & 105 & 104 \\
Stack axial length (mm) & 35 & 36 \\
Air gap (mm) & 0.35 & 0.35 \\
Arc of stator (degree) & 14 & $30.25 / 18.5$ \\
Arc of rotor (degree) & 16 & 30.25 \\
Number of series turns & 5 & 10 \\
Coil span & 3 & 1 \\
Length of coils (mm) & 1878 & $1697(9.63 \% \downarrow)$ \\
Output torque (N.m) & 1.12 & $1.25(11.6 \% \uparrow)$ \\
\hline
\end{tabular}

In order to verify the advantages of the proposed motor, a comparison between it and a conventional 12/8 SRM with the same design dimensions is shown in Table II. It can be seen that the proposed motor can extend $11.6 \%$ of the torque performance while $9.6 \%$ of the copper volume is saved.

\section{EXPERIMENTS ON THE STATIC PERFORMANCE}

The prototype machine has been subjected to static performance testing. Fig. 8 shows the prototype machine with single tooth winding and a segmental rotor. The cores of the rotor and stator are laminated into disk type, and the stator is made up of a single teeth pole, which is wound on the windings, a conventional pole, which is unwound. The cores are embedded into the rotor body which is made up of an aluminum alloy. The aluminum alloy can provide enough mechanical strength, while reducing the weight and rotational inertia of the rotor.

Locked rotor tests were used to determine the flux linkage-current-position characteristics. A DC voltage was applied to one phase and the current was monitored. The flux linkage was then determined using equation (1), and for a constant sampling time, the flux-linkage can be obtained as (2):

$$
\begin{gathered}
\varphi=\int_{0}^{t}(U-i R) d t \\
\varphi_{k+1}=\varphi_{k}+(U-i R) \Delta t
\end{gathered}
$$

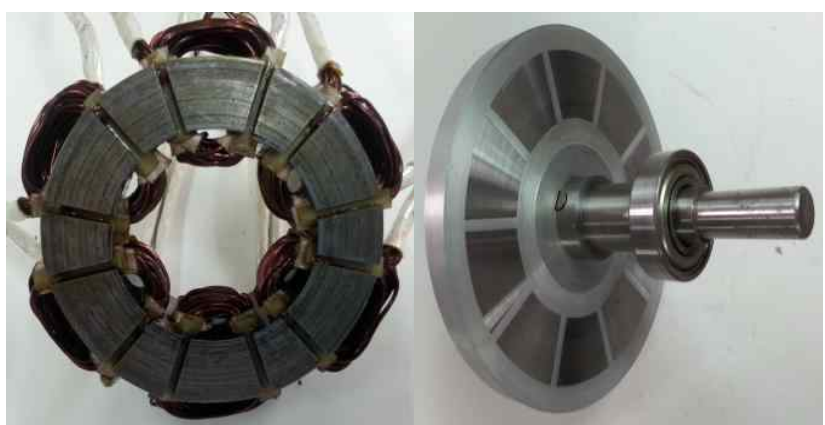

(a) Single teeth winding

(b) Segmental rotor.

Fig. 8. Prototype single teeth stator and segmental rotor

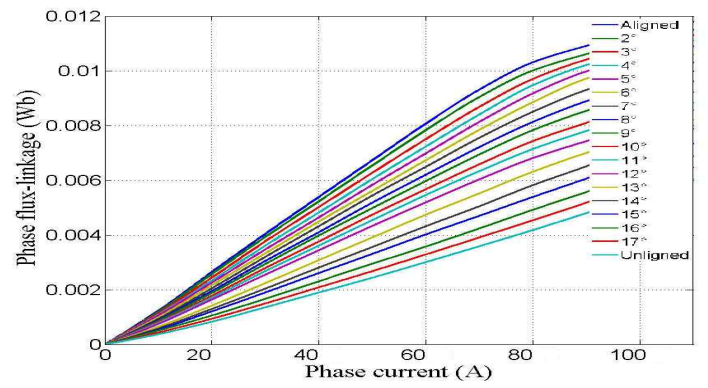

Fig. 9. Measured flux-linkage curves for proposed AF-SRM.

where: $\varphi$ is the flux-linkage; $U$ and $i$ are the measured phase voltage and current, $R$ is the phase resistance, and $\Delta \mathrm{t}$ is the sampling time.

Fig. 9 shows the results of these tests for a series of positions, ranging from the unaligned to the aligned position. For the proposed structure, the rotor pitch is 36 degrees and the motor will be saturated with current. The phase inductance depends on the flux-linkage and current, with half of the rotor pith of 18 degrees from the aligned position to the unaligned position. The inductance curve is reduced with various current levels, and the comparison is shown in Fig. 10. Form this comparison, it can be clearly seen that, the mismatch between the experiment and simulation can be accepted. Fig. 11 shows the static torque performance of the proposed motor and the mismatch between the FEM and the measured results can be accepted.

Fig. 12 shows the control scheme for the drive system. There are two controllers: a current controller and a speed controller. The output of the speed controller is the command current for the current controller. The current limiter block is to keep the command current below the allowable current value. The output of the current controller is the command voltage for the PWM generator.

Fig. 13 shows the experiment setup of the drive system. The load is supplied by a dynamometer and the input power is measured by a power analyzer (PPA2530). The current of the motor is measured by a current sensor (ACS712) and an embedded DSP (TMS320F28335). The DC power is measured 


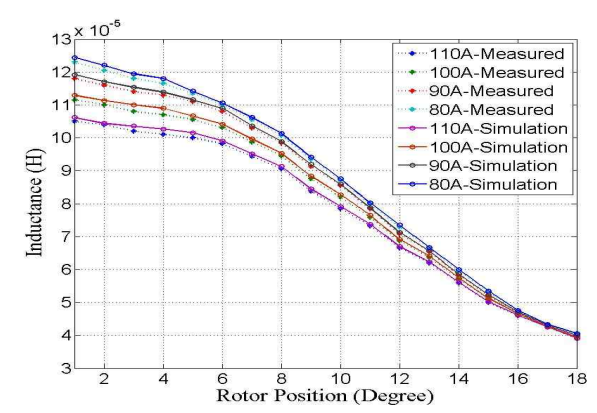

Fig. 10. Inductance profile comparison.

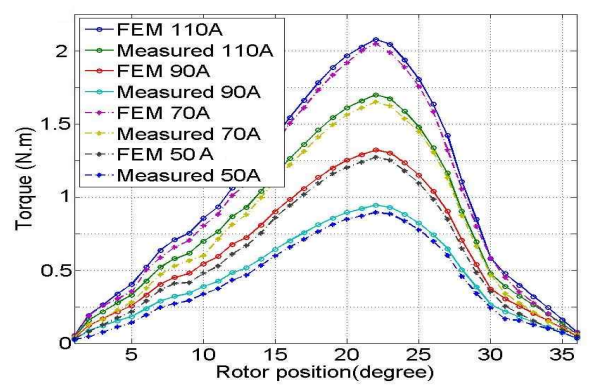

Fig. 11. Torque profile comparison.

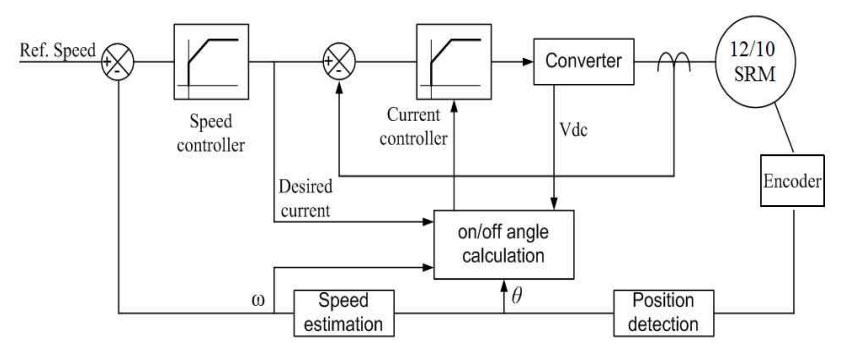

Fig. 12. Control scheme for proposed AF-SRM.

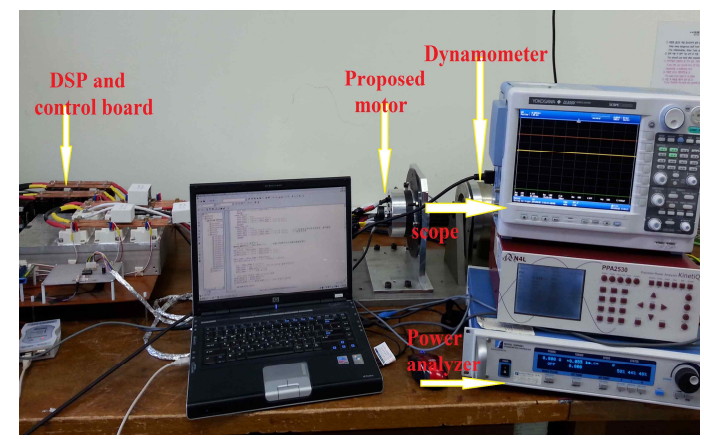

Fig. 13. Experimental setup.

by the scope.

Fig. 14 shows the experimental results of the proposed motor with a full load $1.25 \mathrm{~N} . \mathrm{m}$ at a rated speed of $2800 \mathrm{rpm}$, and for the constant power area with speeds of 2600rpm, $3000 \mathrm{rpm}$ and 3200rpm, respectively. For the constant power area operating, the torque will be reduced while the speed is increased, because the power is equal to the speed multiplied by the torque.

From these results, it can be seen that the measured copper

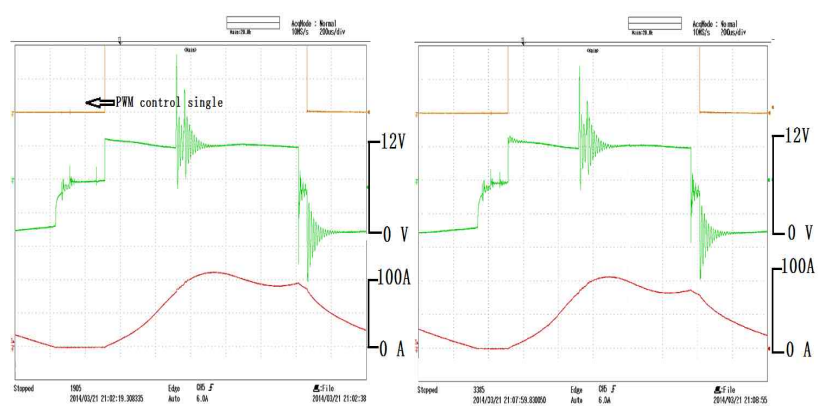

(a) Rated speed $2800 \mathrm{rpm}$. (b) Constant power speed $3200 \mathrm{rpm}$.

Fig. 14. Experimental results of proposed AF-SRM.

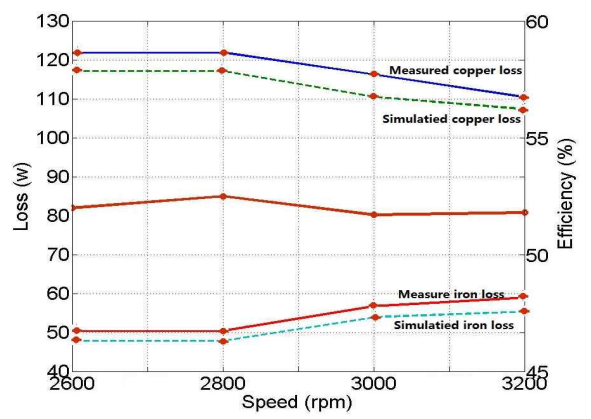

Fig. 15. Measured loss and efficiency results.

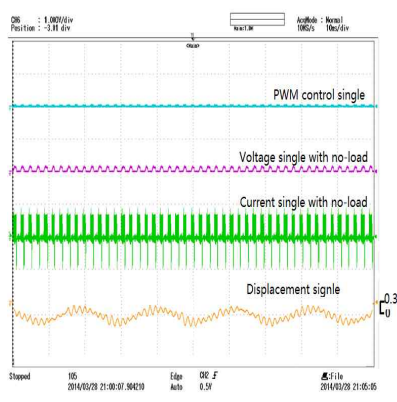

(a) Bottom position.

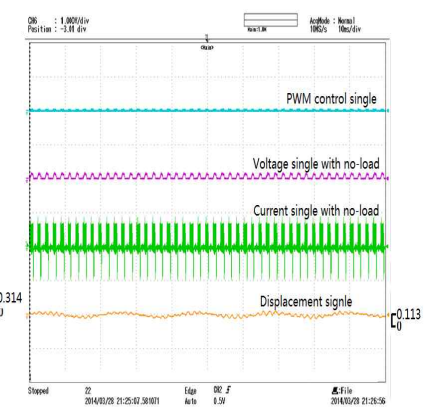

(b) Side position.
Fig. 16. Experimental results of proposed motor.

and iron losses have a total $5 \%$ mismatch with the simulated results. This is caused by the ideal algorithm and the material of the prototype motor. The total efficiency of the motor driver system at its rated value including the power converter and the motor is $53 \%$, as shown in Fig. 15. The output torque is much higher when compare with that of the conventional $12 / 8$, and the copper and iron losses are much smaller.

Vibrations also play an important part during operation of the measurement base on the RION UV-16, with a displacement sensor PV-85. The sensors are fixed on the bottom and side of the stator, and on the top of the rotor. A sensor with the piezoelectric accelerometer method changes the measured signal into a voltage signal. When defining the sensitivity with 80 and the velocity $(\mathrm{mm} / \mathrm{s})$ unit, the experimental result is shown in Fig. 16. With the three different positions it can be seen that the bottom of the stator and the top 
of rotor contain more movement when compared with the side position. The velocity of the bottom of the stator, the top of the rotor and the side of the stator are $0.1041 \mathrm{~mm} / \mathrm{s}, 0.0915 \mathrm{~mm} / \mathrm{s}$ and $0.0339 \mathrm{~mm} / \mathrm{s}$, respectively. Compared with a conventional disk type inductance motor, the vibrations of the proposed motor are at a medium level of displacement.

\section{CONCLUSIONS}

A novel axial field SRM with a single tooth stator and a segmental rotor has been designed, analyzed and compared with a conventional SRM. The axial field design enables a large increase of the flux linking in each turn of the machine, thereby creating a large increase in the torque density. The proposed motor delivers $11.6 \%$ more output torque when compared with a conventional $12 / 8$ SRM with the same dimensions.

The design of a single tooth stator offers an advantage with coils spanning the single teeth, due to the short length of the end-winding. This makes the concept particularly suitable for machines with a relatively short axial length. Because of the single tooth design, $9.63 \%$ of copper volume will be saved when compare with the conventional 12/8 SRM. The concept of the segmental rotor turns the magnetic flux into a short flux path which can reduce the core loss of the rotor. In addition, the segmental cores are embedded into the aluminum body which reduces both the weight of the rotor and the rotational inertia. Finally, simulation analysis and comparison with a conventional SRM are used to verify that the proposed motor is much more effective and suitable for low speed and high torque applications.

There is still some subsequent research that should be conducted, such as the optimization of the axial force, which can be saved by mechanical methods or the double stator one rotor structure. In addition, in terms of the control method, the torque share function will be used to reduce the torque ripple.

\section{ACKNOWLEDGMENT}

This work was supported by the Ministry of Education, through BK21plus.

\section{REFERENCES}

[1] M. Sanada, S. Morimoto, Y. Takeda, and N. Matusi, "Novel rotor pole design of switched reluctance motors to reduce the acoustic noise," IEEE Conference on industry applications, Vol. 1, pp. 107-113, 2000.

[2] C. Lee and R. Krishnan, "New designs of a two-phase e-core machine by optimizing of magnetic structure for a specific application: concept, design, and analysis," IEEE Trans. Ind. Appl., Vol. 45, No. 5, pp. 1804-18014, Sep. 2009.

[3] R. Krishnan and N. S. Lobo, "Apparatus and method that prevent flux reversal in the stator back material of a two-phase SRM," U.S. Patent 7015 615, Mar. 21, 2006.

[4] B. C. Mecrow, J. W. Finch, E. A. El-Kharashi, and A. G.
Jack, "The design of switched reluctance motors with segmental rotors," 15th Int. Conf. on Electrical machines, 2002.

[5] G. A. Horst, "Isolated segmental switched reluctance motor', United States Patent No. 5,111,096, May 5, 1992.

[6] Panu Kurronen, "Torque vibration model of axial-flux surface mounted permanent magnet synchronous machine," Ph.D. dissertation, Lappeenranta University of Technology, Finland, 2003.

[7] M. Aydin, S. Hung, T. A. Lipo, "Design and 3D electromagnetic field analysis of non-slotted and slotted TO-RUS type axial flux surface mounted permanent magnet disc machines," Electric Machines and Drives Conference, 2001. IEMDC 2001. IEEE International, pp. 645-651, 2001.

[8] B. Wang, D.-H. Lee, and J.-W. Ahn, "Characteristic analysis of a novel segmental rotor axial field switched reluctance motor with single teeth winding," in Proc. 15th Int. Conf. IEEE ICIT, pp. 175-180, 2014.

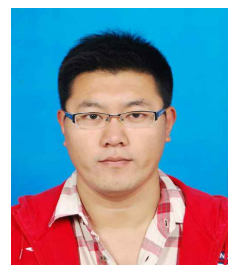

Bo Wang was born in Shenyang, Liaoning, China, in 1985. He received his B.S. and M.S. degrees in Electrical Engineering from the Shenyang University of Technology, Shenyang, China, in 2009 and 2012, respectively. $\mathrm{He}$ is presently working towards his Ph.D. in the Department of Mechatronics Engineering, Kyungsung University, Busan, Korea. His current research interests include SR motor design and SR motor control systems.

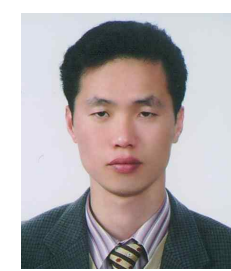

Dong-Hee Lee was born on November 11, 1970. He received his B.S., M.S., and Ph.D. degrees in Electrical Engineering from Pusan National University, Busan, Korea, in 1996, 1998, and 2001, respectively. From 2002 to 2005, he was a Senior Researcher with the Servo R\&D Team, OTIS-LG Company. Since 2005, he has been an Associate Professor with the Department of Mechatronics Engineering, Kyungsung University, Busan, Korea. In 2012, he was a Visiting Professor with the University of Wisconsin-Madison, Madison, WI, USA. He is an Associate Editor of the Journal of Power Electronics. His current research interests include power electronics and motor control systems.

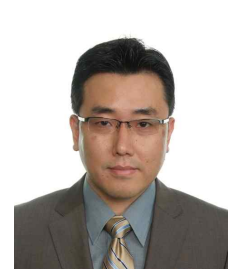

Chee-Woo Lee was born in Busan, Korea, in 1972. He received the B.S. and M.S. degrees in electrical engineering from Pusan National University, Pusan, South Korea, in 1996 and 1998, respectively. He received $\mathrm{PhD}$ in electrical and computer engineering at Virginia Polytechnic Institute and State University, Blacksburg, USA. $\mathrm{He}$ is an assistant professor of electrical engineering at Pusan National University. His research interests include the design of electric machines and their optimal operation.

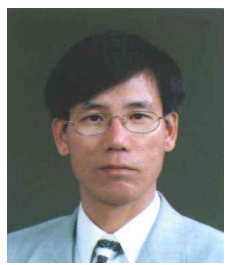

Jin-Woo Ahn was born in Busan, Korea, in 1958. He received his B.S., M.S., and Ph.D. degrees in Electrical Engineering from Pusan National University, Busan, Korea, in 1984, 1986 and 1992, respectively. He has been a Professor in the Department of Mechatronics Engineering, Kyungsung 
University, Busan, Korea, since 1992. He was a Visiting Professor in the Department of Electrical and Computer Engineering and in WEMPEC at the University of Wisconsin-Madison, Madison, WI, USA, from July 1998 to July 1999. He was also a Visiting Professor in the Department of Electrical and Computer Engineering, Virginia Tech, Blacksburg, VA, USA, from July 2006 to June 2007. Professor Ahn is an Adjunct Professor of Shenyang University of Technology, Shenyang, China. He is the director of the Smart Mechatronics Advanced Research and Technology Institute. He is the author of five books including SRM, the author of more than 200 papers and has more than 30 patents. His current research interests include advanced motor drive systems and electric vehicle drives. He has received many awards including a 2011 Busan Science and Technology Award He also received Ministerial Citations from the Ministry of Knowledge Economy and Ministry of Health and Welfare, Korea for his contributions to electrical engineering and industry in 2011 and 2013, respectively. He has served as a Conference Chairman of the International Conference on Electric Machines and Systems 2013 (ICEMS2013), and of the International Conference on Industrial Technology 2014 (IEEE/ICIT2014). He is the Editor-in-Chief of the Journal of International Conference on Electric Machines and Systems (JICEMS). He is a Fellow Member of the Korean Institute of Electrical Engineers, a Member of the Korean Institute of Power Electronics and a Senior Member of the IEEE. 